\title{
ZERO-FREE REGIONS OF LINEAR PARTIAL FRACTIONS*
}

\author{
BY MORRIS MARDEN $\dagger$
}

1. Introduction. The object of this paper is to determine simple regions in the plane which do not contain any zeros of the partial fraction

$$
\Phi=\sum_{j=1}^{n} \frac{\alpha_{j}}{z-a_{j}},
$$

when the $\alpha_{j}$ 's are complex constants.

The case of real $\alpha_{i}$ 's has already been adequately treated by Gauss, Lucas, Jensen, and Bôcher in connection with their study of the derivative of a polynomial and the jacobian of two binary forms. In terms of $\Phi$, their results may be stated as follows.

(a) If all the $\alpha_{j}$ 's have the same sign, there are no zeros of $\Phi$ outside of any convex polygon enclosing the points $a_{j}$. If all the $a_{j}$, in addition, lie on the line-segment $A B$, there are no zeros of $\Phi$ except at points on $A B \ddagger$.

(b) If all the $\alpha_{j}$ 's have the same sign, and the $a_{j}$ 's are either real or in conjugate imaginary pairs, there are no imaginary zeros of $\Phi$ at points outside of all the Jensen circles. $\|$ In any Jensen circle containing $k a_{j}$ 's, and not overlapping any other Jensen circle, there are at least $k-1$ and at most $k+1$ roots of $\Phi . \uparrow$

* Presented to the Society, October 27, 1928.

$\dagger$ National Research Fellow, at Princeton University.

$\ddagger$ The result of which this theorem is an immediate consequence was first stated by Gauss, (Werke, vol. 3, p. 112), in 1816, but rediscovered by Lucas (Comptes Rendus, 1868). This theorem, with reference to the derivative of a polynomial, was first stated by Lucas, Journal de l'École Polytechnique, vol. 46 (1879), p. 8.

\| See $\$ 6$ for definition. This part of the theorem was stated without proof by Jensen, Acta Mathematica, vol. 36 (1912), p. 190; proved by Walsh, Annals of Mathematics, vol. 22 (1920), pp. 128-144.

I Walsh, ibid. 
(c) If $\sum \alpha_{j}=0$ and if all the $a_{j}$ corresponding to positive $\alpha_{j}$ and to negative $\alpha_{j}$ lie respectively in the non-overlapping circular regions $C_{1}$ and $C_{2}$, there are no zeros of $\Phi$ at points outside of these regions.*

2. Method. In the case of complex $\alpha_{j}$ 's as in the case of the real, the zero-free regions of $\Phi$ may be readily obtained when $K(\Phi)$ the conjugate imaginary of $\Phi$, is interpreted as the resultant of all the vectors $\dagger$

$$
f_{j}=\frac{\beta_{i}}{\bar{z}-\bar{a}_{j}} \text { and } g_{j}=-\frac{i \gamma_{j}}{\bar{z}-\bar{a}_{i}} ; \quad(j=1,2, \cdots, n) .
$$

where $\beta_{j}+i \gamma_{j}=\alpha_{j}$. If we set $a_{j}-z=r_{j} e^{i \theta j}$, we may write

$$
f_{j}=\frac{\beta_{i}}{r_{j}} e^{i\left(\theta_{j}+\pi\right)} \text { and } g_{j}=\frac{\gamma_{j}}{r_{j}} e^{i\left(\theta_{j}+\pi / 2\right)} .
$$

These equations tell us that, as vectors drawn from $z$, the first along the line $a_{j} z$ and the second perpendicular to this line, and having magnitudes contingent only upon $r_{j}$ and $\alpha_{j}$, the $f_{j}$ and $g_{j}$ are both independent of choice of axes. A zerofree region of $\Phi$ is then one in which the vector

$$
K(\Phi)=\sum_{j=1}^{n}\left(f_{j}+g_{j}\right)
$$

or its component in any direction does not vanish.

As the $\alpha_{i}$ are here complex numbers, it is convenient to regard them as points in a separate $\alpha$-plane. In this plane it may be assumed without loss of generality, due to the form of $\Phi$, that none of the $\alpha_{j}^{\prime}$ 's lies at the origin. This is equivalent to supposing that, for all $j$,

$$
\left|f_{j}\right|+\left|g_{j}\right|>0 \text {. }
$$

Since multiplication of $\Phi$ by $e^{k i}$ does not affect its zeros, any

* Bôcher, Proceedings of the American Academy, vol. 40 (1904), pp. 469-484.

$\dagger$ The vector $F_{j}=f_{j}+g_{j}$ may be regarded as the force on a unit particle at $z$ exerted according to the inverse distance law by a particle of mass $\alpha_{j}$ at $a_{j}$. See Bôcher, loc. cit., p. 475 , footnote. 
theorem true about the zeros of $\Phi$ for a given distribution $D$ of the $\alpha_{j}$ 's is likewise true for any distribution $D^{\prime}$ obtainable from $D$ by a rotation of the $\alpha$-plane about its origin. Our theorems will however be stated only for simple distributions of the $\alpha_{j}$ 's, the understanding being, of course, that the same theorems hold for somewhat more general distributions.

3. First Application. The simplest cases with complex $\alpha_{j}$ 's may be derived from the theorems of $\$ 1$ by replacing points on the real axis by points on an arbitrary line through the origin. These cases we shall consider, however, by reason of the closing remark of $\$ 2$, as already accounted for.

Let us assume at first instead that all the points $\alpha_{j}$ lie in* the first quadrant of the $\alpha$-plane and the corresponding $a_{j}$ in* the rectangle $B_{1} B_{2} B_{3} B_{4}$.

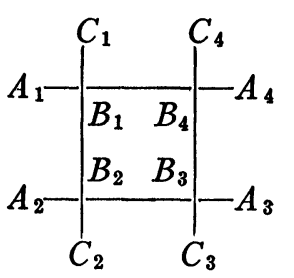

If $z$ is any point in the angle $A_{4} B_{4} C_{4}$, the components of $f_{k}$ and $g_{k}$ parallel to $A_{1} A_{4}$ and $C_{1} C_{2}$ will be, respectively,

$$
\begin{aligned}
& H_{k}=\left|f_{k}\right| \cos \phi_{k}+\left|g_{k}\right| \sin \phi_{k}, \\
& V_{k}=\left|f_{k}\right| \sin \phi_{k}-\left|g_{k}\right| \cos \phi_{k},
\end{aligned}
$$

$\phi_{k}$ being the angle between $f_{k}$ and a parallel to $A_{1} A_{4}$.

In order to locate the zeros of $\Phi$ in the angle $A_{4} B_{4} C_{4}$, we shall try to find points at which $\sum_{1}^{n} H_{k}=\sum_{1}^{n} V_{k}=0$.

As $0 \leqq \phi_{k} \leqq \pi / 2, H_{k} \geqq 0$. Because of (1), $H_{k}=0$ for all $k$ only in the following cases:

I.

$$
f_{k}=\phi_{k}=0, \quad \text { all } k
$$

II.

$$
g_{k}=\phi_{k}-\frac{\pi}{2}=0, \text { all } k \text {; }
$$

III. $f_{k}=\phi_{k}=0$, some $k, g_{k}=\phi_{k}-\pi / 2=0$, remaining $k$.

In cases I and II, no zeros of $\Phi$ turn up because the $V_{k}$ are of the same sign and therefore $\sum_{1}^{n} V_{k} \neq 0$.

In case III, on the contrary, where some of the $a_{j}$ lie on

* In $\S \S 3,4,5$, we shall use all our locative phrases "in," "to the right of," etc. in the wide sense, including thereby the boundary points of the regions considered. In $\$ \$ 6$ and 7 , we shall revert to the strict sense of these terms. 
the line $B_{1} B_{4}$ and the corresponding $\alpha_{j}$ on the imaginary axis, and the remaining $a_{j}$ lie on the line $B_{3} B_{4}$ and the corresponding $\alpha_{j}$ on the real axis, there is a possibility of $\sum_{1}^{n} V_{k}=0$ and of a zero of $\Phi$ at the point $B_{4}$.

Similar considerations hold in the remaining angles $A_{k} B_{k} C_{k}$. There are no zeros of $\Phi$ in any of the angles $A_{k} B_{k} C_{k}$, except perhaps at just one of the points $B_{k}$. If, furthermore, all the $a_{k}$ lie on $B_{1} B_{4}$, there are no zeros to the right of $C_{3} C_{4}$ or to the left of $C_{1} C_{2}$.

4. Corollaries. More elegant results for the case that the $\alpha_{j}$ 's are in the first quadrant can now be obtained if the corresponding $\alpha_{j}$ are required to lie in a circle $C$ of radius $R$. In the concentric circle $C^{\prime}$ of radius $2^{1 / 2} R$, a square $S$ can be inscribed which will circumscribe $C$ and which, when suitably chosen, will include in an angle $A_{k} B_{k} C_{k}$ any point outside $C^{\prime}$. Consequently there are no zeros of $\Phi$ outside of $C^{\prime}$.

If $n=2$, "outside of $C^{\prime \prime}$ must be taken in the strict sense. If $a_{1}$ and $a_{2}$ are situated on $C, \pi R / 2$ units apart, $S$ may have $a_{1}$ on one side and $a_{2}$ on the adjacent side. Case III of $\S 3$ would then permit a zero to be on $C^{\prime}$, as the example

$$
\frac{i}{z-i}+\frac{1}{z-1}=0
$$

with the unit circle as $C$, shows.

If the points $a_{j}$ just considered are all on the line-segment $A B, \Phi$ will have a larger zero-free region. On $A B$ as diagonal a rectangle $R$ can so be constructed as to include in an angle $A_{k} B_{k} C_{k}$ any point outside of the circle $\Gamma$ having $A B$ as diameter. Outside of $\Gamma$ there are therefore no zeros of $\Phi$.

Again "outside of $\Gamma$ " must be given a strict meaning when $n=2$. If $a_{1}$ and $a_{2}$ are at $A$ and $B$, case III arises with its possibility of a zero on $\Gamma$, as for instance in the example

$$
\frac{1}{z-1}+\frac{i}{z+1}=0
$$

where $A:(-1,0)$ and $B:(1,0)$. 
5. Other Applications. By essentially the methods of $\S 3$, the following results may also be deduced:

(a) Suppose the $\alpha_{j}$ to consist of two groups $G_{1}$ and $G_{2}$, the former situated in the first quadrant and the latter in the second quadrant of the $\alpha$-plane. If the $a_{j}$ corresponding to $G_{1}$ and $G_{2}$ lie respectively in the angles $A_{1} B_{1} C_{2}$ and $A_{4} B_{4} C_{3}$, there are no zeros of $\Phi$ in the strip $C_{1} B_{1} B_{4} C_{4}$, save perhaps upon the segment $B_{1} B_{4}$.

The exceptions may occur only if $G_{1}=G_{1 a}+G_{1 c}$ and $G_{2}=G_{2 a}+G_{2 c}$, where $G_{1 a}$ and $G_{2 a}$ have their $\alpha_{j}^{\prime}$ 's on the imaginary axis, $G_{1 c}$ and $G_{2 c}$ their $\alpha_{j}^{\prime}$ 's on the real axis, and $G_{1 a}, G_{1 c}, G_{2 a}$, and $G_{2 c}$ have their $a_{j}$ 's respectively on the segments $A_{1} B_{1}, B_{1} C_{2}, A_{4} B_{4}, B_{4} C_{3}$.

If $G_{1 c}=G_{2 c}=0$ (that is, $G_{1 c}$ and $G_{2 c}$ contain no points), any point of $B_{1} B_{4}$ may be a zero of $\Phi$.

If $G_{1 a} \cdot G_{1 c} \neq 0$ and $G_{2 c}=0$, the point $B_{1}$ is the only possible zero of $\Phi$ on $B_{1} B_{4}$.

If $G_{1 c}=0$ and $G_{2 a} \cdot G_{2 c} \neq 0$, the point $B_{4}$ is the only possible zero of $\Phi$ on $B_{1} B_{4}$.

If the lines $C_{1} C_{2}$ and $C_{3} C_{4}$ are coincident, we shall exclude the case $G_{1 a}=G_{2 a}=0$, but include the cases

and

$$
\begin{aligned}
& G_{1 a}=0 \text { and } G_{2 a} \cdot G_{2 c}=0, \\
& G_{2 a}=0 \text { and } G_{1 a} \cdot G_{1 c}=0,
\end{aligned}
$$

$$
G_{1 a} \cdot G_{1 c} \cdot G_{2 a} \cdot C_{2 c} \neq 0 \text {, }
$$

in which cases $B_{1}$ is the only possible zero of $\Phi$ on $B_{1} B_{4}$.

(b) Suppose the $\alpha_{j}$ to consist of four groups $G_{k}(k=1,2,3,4)$, the group $G_{k}$ being in the kth quadrant of the $\alpha$-plane. If the $a_{j}$ corresponding to $G_{k}$ lie in the angle $A_{k} B_{k} C_{k}$, there are no zeros of $\Phi$ in the rectangle $B_{1} B_{2} B_{3} B_{4}$.

We are supposing no two sides of $B_{1} B_{2} B_{3} B_{4}$ to coincide. A degenerate case of interest is, however, one in which $A_{1} A_{4}$ and $A_{2} A_{3}$ are coincident and the $a_{j}$ corresponding to $G_{k}$ lie on $A_{1} B_{1}$ if $k=1$ or 2 , and on $A_{4} B_{4}$ if $k=3$ or 4 . Then, if not all the $\alpha_{j}$ 's lie on the real axis, there are no zeros of $\Phi$ on the segment $B_{1} B_{4}$. The theorems of (a) and (b) also hold for $n=\infty$ provided $\Phi$ is absolutely convergent in the abovementioned zero-free regions. 
6. A Jensen Case. So far no restrictions have been placed upon the behavior of $\Phi$ for real values of $z$. In this section we shall assume, in order to make $\Phi$ real on the real axis, that the $a_{j}$ and the corresponding $\alpha_{j}$ are both real or both in conjugate imaginary pairs. We shall also suppose the $\alpha_{i}$ corresponding to an $a_{j}$ in the upper half-plane also to be in the upper halfplane, and vice-versa. Furthermore, we shall limit the $\alpha_{j}$ to the first and fourth quadrants of the $\alpha$-plane.*

Let us construct in the z-plane the circles (the Jensen circles) having as diameters the line segments joining pairs of conjugate $a_{j}$, and the indefinite lines (the Jensen lines) through the pairs of conjugate $a_{j}$. If we denote by $R$ the region consisting of all points simultaneously outside of all the Jensen circles and to the left of all the Jensen lines, we may define $R$ by the inequalities

$$
\left(x-b_{j}\right)^{2}+y^{2}-c_{j}^{2}>0, \quad x-b_{j}<0 ;
$$

all $j$ corresponding to imaginary $a_{j}=b_{j}+i c_{j}$.

In the sum $\Phi$ let us discern two types of terms, one corresponding to real $a_{j}$ :

$$
t_{1}=\frac{\beta_{i}}{z-b_{j}}=\frac{\beta_{j}\left[\left(x-b_{j}\right)-i y\right]}{\left(x-b_{j}\right)^{2}+y^{2}}, \quad\left(\alpha_{j}=\beta_{i}+\gamma_{j}\right),
$$

and the other corresponding to a pair of conjugate $a_{j}$ :

$$
t_{2}=\frac{\beta_{j}+i \gamma_{j}}{z-\left(b_{j}+i c_{j}\right)}+\frac{\beta_{j}-i \gamma_{j}}{z-\left(b_{j}-i c_{j}\right)} .
$$

The latter has as component parallel to the $y$-axis:

$$
I\left(t_{2}\right)=\frac{-2 y\left[\beta_{j}\left\{\left(x-b_{j}\right)^{2}+y^{2}-c_{j}{ }^{2}\right\}-2 c_{j} \gamma_{j}\left(x-b_{j}\right)\right]}{\left\{\left(x-b_{j}\right)^{2}+\left(y-c_{j}\right)^{2}\right\}\left\{\left(x-b_{j}\right)^{2}+\left(y+c_{j}\right)^{2}\right\}} .
$$

As $\beta_{j} \geqq 0, c>0$, and $\gamma>0$, we find in $R$

$$
\operatorname{sgn} I\left(t_{1}\right)=\operatorname{sgn} I\left(t_{2}\right)=-\operatorname{sgn} y .
$$

The components of $t_{1}$ and $t_{2}$ parallel to the $y$-axis will there-

* See the footnote to $\$ 3$. 
fore not vanish at points in $R$ off the real axis. That is to say, there are no imaginary roots of $\Phi$ in $R$.

The question of the real roots of $\Phi$ in $R$ may be easily settled through a study of the real function $\Phi$ on the real axis. Again we shall separate the terms corresponding to real $a_{j}$ from those corresponding to pairs of conjugate $a_{j}$. The first

$$
S_{1}=\frac{\beta_{j}}{x-b_{j}} \quad \text { has } \quad \frac{d S_{1}}{d x}=-\frac{\beta_{j}}{\left(x-b_{j}\right)^{2}} ;
$$

the second

$$
\begin{aligned}
S_{2} & =\frac{\beta_{j}+i \gamma_{j}}{x-\left(b_{j}+i c_{j}\right)}+\frac{\beta_{j}-i \gamma_{j}}{x-\left(b_{j}-i c_{j}\right)}, \\
\frac{d S_{2}}{d x} & =\frac{2 \beta_{j}\left\{c_{j}{ }^{2}-\left(x-b_{j}\right)^{2}\right\}+4 c_{j} \gamma_{j}\left(x-b_{j}\right)}{\left\{\left(x-b_{j}\right)^{2}+c_{j}{ }^{2}\right\}^{2}} .
\end{aligned}
$$

Therefore $d \Phi / d x<0$ in $R$. In addition, as $x$ moves to the right from one $a_{j}$ to the next $a_{j}$, $\Phi$ changes from $+\infty$ to $-\infty$. In other words, any interval of the real axis in $R$ not containing any $a_{j}$ has at most one root of $\Phi$; between two successive $a_{j}$ in $R$, there is exactly one root of $\Phi$. In short, if the number of $a_{j}$ in $R$ is $k$, the number of real roots of $\Phi$ in $R$ is $k$, $k-1$, or $k+1$.* $^{*}$

The above equations also allow us to deduce at once the results for the case in which the $\alpha_{j}$ corresponding to real $a_{j}$ lie on the positive real axis, and the remaining $\alpha_{j}$ are in the second and third quadrants of the $\alpha$-plane. The above theorems will hold for this case if $R$ in these theorems is replaced by $K$, the region consisting of all points simultaneously inside of all the Jensen circles and to the left of all the Jensen lines. ( $K$ may not contain any points.)

These theorems will also be valid when $n=\infty$ provided $\Phi$ converges uniformly in any closed region in $R$ or $K$ not including any points $a_{j}$, and all the imaginary $a_{j}$ lie to the right of a fixed line parallel to the $y$-axis.

*E. B. Van Vleck has an unpublished proof of Jensen's theorem which uses similar analysis. 
7. The Function $\eta(z)$. As a final application particularly of the methods of $\S 6$, we turn to the function

$$
\begin{aligned}
\eta(z) & =\frac{1}{z}\left\{\frac{\zeta^{\prime}(z)}{\zeta(z)}-(1+\log 2 \pi)\right\} \\
& =\frac{2}{z^{2}}-\frac{1}{z-1}-\sum_{k=1}^{\infty} \frac{1}{k(z+2 k)}+\sum_{-\infty}^{\infty} \frac{1}{\rho_{n}\left(z-\rho_{n}\right)},
\end{aligned}
$$

where $\zeta(z)$ is the Riemann zeta function, and the $\rho_{n}$ its zeros.

Concerning the $\rho_{n}$, it is known* that

$$
\begin{array}{cr}
\rho_{-n}=\bar{\rho}_{n} & \\
0<R\left(\rho_{n}\right)<1, & \quad \text { (all } n), \\
R\left(\rho_{n}\right)=\frac{1}{2}, & \text { for }\left|I\left(\rho_{n}\right)\right| \leqq 300,
\end{array}
$$

and that $\rho=\frac{1}{2} \pm K i, K=14.13472(5)$, are the nearest $\rho_{n}$ to the real axis.

Let us draw a circle of radius $K$, center at $(1 / 2,0)$ and denote by $S$ the interior of this circle to the left of the imaginary axis. Then in $S$ there are no imaginary zeros of $\eta(z)$. In each of the intervals $(0,-2),(-2,-4),(-4,-6),(-6,-8)$, $(-8,-10)$, and $(-10,-12)$ of the real axis there is exactly one zero, and in the interval $\left(-12, \frac{1}{2}-K\right)$ there is at most one zero of $\eta(z)$.

The University of Wisconsin

* See Hutchinson, Transactions of this Society, vol. 27 (1925), p. 49, and references therein. My attention was called to the logarithmic derivative of the zeta function by C. E. Hille. 